LA-UR-01-5242

Approved for public release: distribution is unlimited.

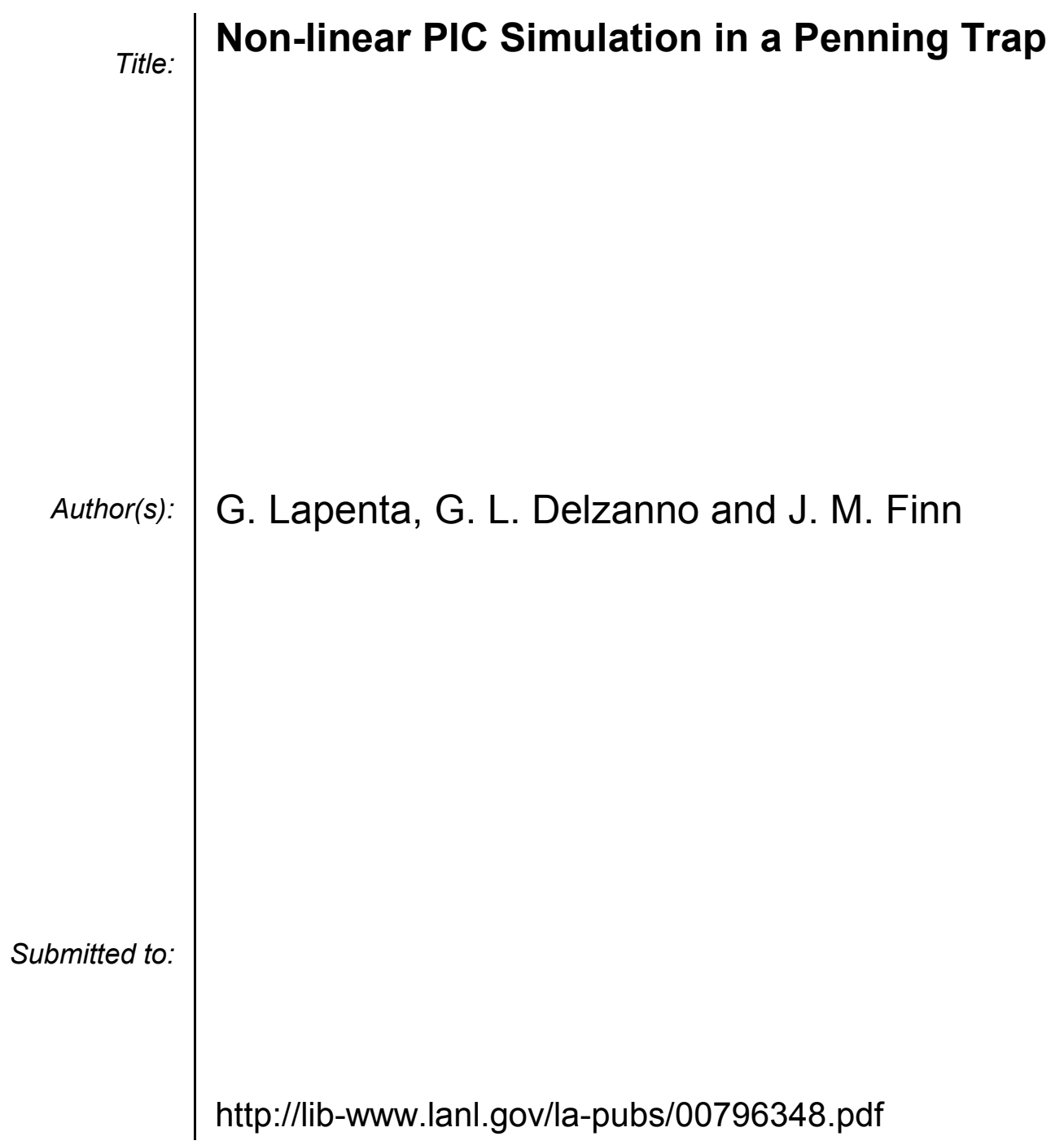

Los Alamos National Laboratory, an affirmative action/equal opportunity employer, is operated by the University of California for the U.S. Department of Energy under contract W-7405-ENG-36. By acceptance of this article, the publisher recognizes that the U.S. Government retains a nonexclusive, royaltyfree license to publish or reproduce the published form of this contribution, or to allow others to do so, for U.S. Government purposes. Los Alamos National Laboratory requests that the publisher identify this article as work performed under the auspices of the U.S. Department of Energy. Los Alamos National Laboratory strongly supports academic freedom and a researcher's right to publish; as an institution, however, the Laboratory does not endorse the viewpoint of a publication or guarantee its technical correctness. 


\title{
Non-linear PIC Simulation in a Penning Trap
}

\author{
G. Lapenta*, G. L. Delzanno ${ }^{\dagger}$ and J. M. Finn** \\ ${ }^{*}$ Istituto Nazionale per la Fisica della Materia (INFM) and Theoretical Division, Los Alamos \\ National Laboratory, Los Alamos, NM 87545, USA \\ ${ }^{\dagger}$ Istituto Nazionale di Fisica per la Materia (INFM) and Dipartimento di Energetica, Politecnico di \\ Torino, Corso Duca degli Abruzzi 24, 10129 Torino, Italy \\ ** Theoretical Division, Los Alamos National Laboratory, Los Alamos, NM 87545, USA
}

\begin{abstract}
We study the non-linear dynamics of a Penning trap plasma, including the effect of the finite length and end curvature of the plasma column. A new cylindrical PIC code, called KANDINSKY, has been implemented by using a new interpolation scheme. The principal idea is to calculate the volume of each cell from a particle volume, in the same manner as it is done for the cell charge. With this new method, the density is conserved along streamlines and artificial sources of compressibility are avoided. The code has been validated with a reference Eulerian fluid code. We compare the dynamics of three different models: a model with compression effects, the standard Euler model and a geophysical fluid dynamics model. The results of our investigation prove that Penning traps can really be used to simulate geophysical fluids.
\end{abstract}

\section{INTRODUCTION}

Malmberg-Penning traps are often characterized as a tool to simulate, in a plasma, the 2D dynamics described by the Euler equations. However, it is well known that the classical 2D drift-Poisson model (mathematically equivalent to the 2D Euler model [1]) is unable to capture the features of the $k=1$ diocotron instability. In this approximation, there are no unstable modes [2] and the continuum spectrum can give only algebraic growth [3]; instead, experiments show an exponential growth of the mode [4].

Recently, the research has focused on trying to resolve this contradiction between theory and experiments. Finn et al. [5] were the first to explain the instability in terms of effects due to the finite length and end curvature of the plasma column (compression effects) and later Coppa et al. [6] improved this fluid model by modifying the expression for the velocity field, by including temperature effects and by using the exact Green's function for the plasma length. Hilsabeck and O'Neil [7] pointed out that kinetic effects might also be important in the $k=1$ diocotron instability.

Interestingly, when compression effects are taken into account, the classical analogy between the 2D drift-Poisson model and the Euler model for an incompressible and inviscid fluid breaks down. Instead, a new analogy between non-neutral plasmas and geophysical fluid dynamics (GFD) arises [5, 8], in which the plasma length plays the role of the inverse of the fluid depth. This analogy is very important from the point of view of performing geophysical experiments in Penning traps.

In the present contribution, we focus on a simplified version of the model presented in Ref. [6], obtained by neglecting the temporal variation of the plasma length. As shown in Ref. [6], this model is still $k=1$ unstable. The model is compared with the Euler model and with the $\gamma$-plane approximation of the geophysical fluid dynamics.

We present simulations of the non-linear dynamics obtained with the cylindrical PIC Code KANDINSKY, showing that GFD can be actually simulated in Penning traps. 


\section{PHYSICAL MODEL}

A cylindrical Malmberg-Penning trap confining an electron plasma is considered in the following. The trap consists of three electrodes. The central cylinder, which extends between $z=-L_{c} / 2$ and $z=L_{c} / 2$, is grounded, while the two cylindrical end caps are at a negative potential, $-V$. An uniform magnetic field provides radial confinement, while the potential difference between the central electrode and the end caps provides axial confinement.

In typical experiments, the electron cyclotron radius is much smaller than the typical size of the device, allowing the guiding center approximation. Furthermore, the dependence of the plasma properties along the axial direction can be simplified considerably thanks to the rapid bouncing motion between the end caps. However, a correct description of the diocotron instability, especially for the $k=1$ mode, requires one to include the effect of finite length of the plasma in the axial direction and end curvature.

Following the approach in Refs. [5,6], the particles are described by strings of variable length, which change their axial length and, consequently, their density, if they move radially due to the $\mathbf{E} \times \mathbf{B}$ drift. The effective axial length is calculated selfconsistently by solving the Poisson equation in the trap and assuming thermodynamic equilibrium along the axial direction [9]. In normalized units, the model consists of the following system of equations [6]:

$$
\left\{\begin{array}{l}
\frac{\partial \sigma}{\partial t}=-\mathbf{V}_{\perp} \cdot \nabla_{\perp} \sigma \\
\mathbf{V}_{\perp}=\widehat{\mathbf{e}}_{z} \times \nabla_{\perp} \phi_{\text {eff }} \\
\mathcal{L}=\int \exp \left(\frac{\Psi}{\alpha}\right) d z \\
\nabla^{2} \Psi=\frac{\sigma}{\mathcal{L}}\left[\exp \left(\frac{\Psi}{\alpha}\right)-1\right] \\
\nabla_{\perp}^{2} \phi_{c}=\frac{\sigma}{\mathcal{L}} \\
\phi_{\text {eff }}=\phi_{c}+\alpha \log \frac{\mathcal{L}}{\mathcal{L}_{r=0}}
\end{array}\right.
$$

where $\sigma(r, \theta, t)=\frac{e R_{w}}{\varepsilon_{0} \widehat{\phi}_{c 0}(0)} \widehat{\sigma}$ is the density integrated along axial direction, $\mathbf{V}_{\perp}(r, \theta, t)=$ $\frac{B_{0} R_{w}}{\widehat{\phi}_{c 0}(0)} \widehat{\mathbf{V}}_{\perp}$ is the plasma velocity in the plane $(r, \theta), \mathcal{L}(r, \theta, t)=\frac{\widehat{L}}{R_{w}}$ is the effective length of the plasma, $\Psi(r, \theta, z, t)=\frac{\widehat{\phi}(r, \theta, z, t)-\widehat{\phi}_{c}(r, \theta, t)}{\widehat{\phi}_{c 0}(0)}$ is the correction to the potential in a trap of finite length with respect to the value in a trap of infinite length, $\phi_{\mathrm{eff}}(r, \theta, t)$ is the effective potential, $\alpha=\frac{\Theta}{\widehat{\phi}_{c 0}(0)}$ is the electron temperature, $t=\frac{\widehat{\phi}_{c 0}(0)}{B_{0} R_{w}^{2}} \widehat{t}$ is the time and $R_{w}$ is the wall radius. Hatted quantities are physical and have dimensions; the corresponding normalized quantities share the same symbol but are not hatted. Subscripts $c$ and 0 label, respectively, quantities evaluated in the central plane and unperturbed quantities. For further details on the model and its derivation we refer to Refs. [6] and [10].

The model can be regarded as quasi-2D since only the potential in the trap depends on the axial coordinate and can be reduced to a fully $2 \mathrm{D}$ model by neglecting the time variation of the effective plasma length (in this case one does not need to solve the 3D Poisson equation). In this approximation the new model becomes: 


$$
\left\{\begin{array}{l}
\frac{\partial}{\partial t}\left(n_{c} \mathcal{L}_{0}\right)=-\mathbf{V}_{\perp} \cdot \nabla_{\perp}\left(n_{c} \mathcal{L}_{0}\right) \\
\mathbf{V}_{\perp}=\widehat{\mathbf{e}}_{z} \times \nabla_{\perp} \phi_{\mathrm{eff}} \\
\nabla_{\perp}^{2} \phi_{c}=n_{c} \\
\phi_{\mathrm{eff}}=\phi_{c}+\alpha \log \frac{\mathcal{L}_{0}}{\mathcal{L}_{0, r=0}}
\end{array}\right.
$$

where we used the fact that $\sigma=n_{c} \mathcal{L}_{0}$ and $\mathcal{L}_{0}(r)$ is obtained from the initial equilibrium solution.

Compression effects due to the finite length of the plasma are included in the first and second equation of system (2). In the limit of $\alpha=0$ and $\mathcal{L}_{0} / \mathcal{L}_{0, r=0}=1-s r^{2}$ the model described by Eqs. (2) reduces to the model proposed by Finn et al. (Eq. (38), Ref. [5]a).

In the following we will focus our attention on model (2), since it is simplified but still able to capture the features of the $k=1$ diocotron instability (as shown in Ref. [6]). The spectrum of eigenvalues of model (2) is investigated in Ref. [11].

Our aim is to compare the dynamics of model (2) with the standard Euler model:

$$
\left\{\begin{array}{l}
\frac{\partial n_{c}}{\partial t}=-\mathbf{V}_{\perp} \cdot \nabla_{\perp} n_{c} \\
\mathbf{V}_{\perp}=\widehat{\mathbf{e}}_{z} \times \nabla_{\perp} \phi_{c} \\
\nabla_{\perp}^{2} \phi_{c}=n_{c}
\end{array}\right.
$$

and with the geophysical fluid dynamics (GFD) model [12]:

$$
\left\{\begin{array}{l}
\frac{\partial}{\partial t}\left(\frac{\xi+2 \Omega}{h}\right)=-\mathbf{V}_{\perp} \cdot \nabla_{\perp}\left(\frac{\xi+2 \Omega}{h}\right) \\
\mathbf{V}_{\perp}=\widehat{\mathbf{e}}_{z} \times \nabla_{\perp} \Psi \\
\nabla_{\perp}^{2} \Psi=\xi
\end{array}\right.
$$

where $\xi$ is the vorticity, $\Omega$ is the rotation frequency, $h$ is the height of the free surface of the fluid relative to the bottom topography, $\Psi$ is the streamfunction and $(\xi+2 \Omega) / h$ is the potential vorticity. Model (4) is obtained from the shallow-water equations in the limit of small Rossby number, namely in the quasi-geostrophic approximation. As pointed out in Ref. [5], with $\Omega=0$ the effective length of the plasma $\mathcal{L}_{0}$ plays in model (2) the same role played by $1 / h$ in GFD. It is this analogy which leads to the possibility of simulating experimentally geophysical fluid dynamics in a Penning trap.

In all the models, a quantity is advected by the velocity field: the density (vorticity) $n_{c}$ for Euler, the line integrated density $n_{c} \mathcal{L}_{0}$ for model (2) and the potential vorticity for GFD. All these models have the same equation for the velocity field, but only for the Euler case is the velocity field directly calculated from the quantity which is advected. In model (2) the velocity field is calculated from the solution of the Poisson equation plus an extra term which retains curvature and thermal effects, while in GFD the velocity field is determined by the vorticity $\xi$.

\section{PIC CODE}

The PIC code KANDINSKY has been developed based on a standard cylindrical PIC code previously developed [13] by the authors in collaboration with Gianni Coppa 
and Antonio D'Angola at the Politecnico di Torino. A cylindrical PIC code has many advantages: very low viscosity; boundary conditions can be imposed exactly; the Fast Fourier Transform (FFT) can be used to solve the Poisson equation resulting in an improvement of the speed of the code. Nevertheless, it is well known that cylindrical PIC codes can potentially suffer from a number of problems. Of particular importance to the present study are the noise that typically arises in the center of the system, the nonconservation of enstrophy, and the growth of the maximum value of the density during simulations (inconsistent with incompressible flows). In the following subsections we will show how to improve the cylindrical PIC scheme and to solve the problem.

\section{The problem}

In order to understand the origin of the problems that affect cylindrical PIC codes, we will start with a simple exercise. Consider the 1D uniform cartesian grid of Fig. 1a and consider one particle of unit charge located at positions A, B or C. It is possible to calculate the density of each cell with the volume-weighted (Cloud-In-Cell) interpolation scheme, which is the most common scheme adopted in PIC codes. When the particle is in position $A$, it contributes only to the first cell and the density is $\rho_{1}=1 / \Delta x(\Delta x$ is the grid spacing); when the particle is in position $\mathrm{B}$, it is spread equally on the two cells and $\rho_{1}=\rho_{2}=1 /(2 \Delta x)$; when the particle is in position $\mathrm{C}$, it contributes only to the second cell and $\rho_{2}=1 / \Delta x$. During the motion of the particle from $\mathrm{A}$ to $\mathrm{C}$, the density at the particle position fluctuates between $1 /(2 \Delta x)$ and $1 / \Delta x$ but on average it is conserved over long Lagrangian trajectories. Furthermore, this fluctuation is reduced when summed over the number of particles per cell, and this source of noise is manageable.

We now repeat the same exercise for the uniform cylindrical grid of Fig. 1b. In general a particle would contribute to two cells in $\theta$ and two cells in $r$ direction, since the grid is 2D. In this specific example, we place $A, B$ and $C$ at the same azimuthal angle $\Delta \theta / 2$, so there is only contribution to one cell in $\theta$ and the problem can be considered $1 \mathrm{D}$. When the particle is in position $A$, it contributes only to the first cell and $\rho_{1}=2 /\left(\Delta r^{2} \Delta \theta\right)(\Delta r$ and $\Delta \theta$ are, respectively, the grid spacing in $r$ and $\theta$ directions); when the particle is in position $\mathrm{B}$, its charge is spread equally between the two cells but the different volume of the two cells leads to different densities, $\rho_{1}=1 /\left(\Delta r^{2} \Delta \theta\right)$ and $\rho_{2}=1 /\left(3 \Delta r^{2} \Delta \theta\right)$. When the particle is in position $C$, it contributes only to the second cell and $\rho_{2}=2 /\left(3 \Delta r^{2} \Delta \theta\right)$. Again during the motion from $\mathrm{A}$ to $\mathrm{C}$ the density is transferred from the first cell to the second, but in this case the total density of the particle does not remain constant, even on average. In particular, the density decreases as the particle moves away from the center of the system and this means that the CIC interpolation scheme, applied to a cylindrical grid with uniform spacings $\Delta r$ and $\Delta \theta$, intrinsically violate the incompressibility condition. This effect is partially compensated for on average because more particles are in the cell of volume $r \Delta r \Delta \theta$ for large $r$.

This simple exercise points out that, when dealing with non-uniform cell volumes, standard interpolation schemes produce fictitious compressibility.

We emphasize that this problem affects not only the cylindrical geometry but it occurs whenever a grid of non-uniform volume is used. 

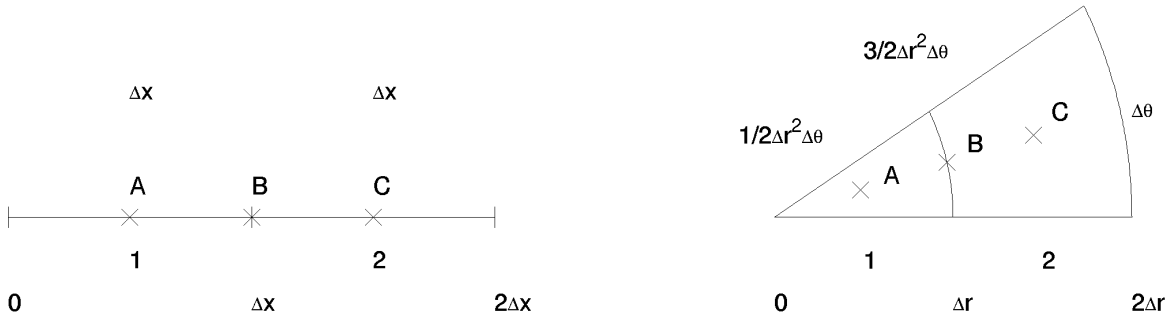

FIGURE 1. a) Uniform cartesian grid (left); b) Uniform cylindrical grid (right).

\section{The solution}

According to [14], we define natural coordinates, $\vec{\omega}=(\zeta, \eta)$ by mapping each cell of the physical grid onto a unit square cell in the space of natural coordinates. Therefore, in the space of natural coordinates the grid is uniform and cartesian. We define a shape function $s\left(\vec{\omega}-\vec{\omega}_{p}\right)$ in order to interpolate from the particles to the grid and viceversa, normalized to $\iint s d \zeta d \eta=1$ in the space of natural coordinates. Then, the shape function in the physical space is $S\left(\mathbf{x}(\vec{\omega})-\mathbf{x}_{p}\left(\vec{\omega}_{p}\right)\right)=s\left(\vec{\omega}-\vec{\omega}_{p}\right)$. When the grid and the particles move with the fluid velocity and the interpolation scheme is consistent with the choice of the grid, it can be shown that the natural coordinates are constants of the motion [14]; in other words, $s\left(\vec{\omega}-\vec{\omega}_{p}\right)$ is constant during the Lagrangian phase in the space of natural coordinates.

In order to solve the problem that we described in the previous paragraph, we introduce the volume associated with each particle, $V_{p}$, in the same manner as it is done for the particle charge $q_{p}$, and to calculate the volume of each cell we use the same interpolation scheme adopted for the charge:

$$
V(\vec{\omega})=\sum_{p} V_{p} w\left(\vec{\omega}-\vec{\omega}_{p}\right)
$$

where $w$ is the assignment function associated with the shape function $s$ [15]. Then the density is given by:

$$
\rho(\vec{\omega})=\frac{\sum_{p} q_{p} w\left(\vec{\omega}-\vec{\omega}_{p}\right)}{\sum_{p} V_{p} w\left(\vec{\omega}-\vec{\omega}_{p}\right)} .
$$

This definition gives conservation of $\rho$ along the Lagrangian trajectories of each particle, since $q_{p}$ and $V_{p}$ are constant (determined at the beginning of each simulation) and each particle is advected with constant $w$ in the space of natural coordinates.

\section{PIC summary}

The KANDINSKY code consists of four parts: the loading of particles, the interpolation scheme, the Poisson solver and the particle mover. The implementation of each 
part of the code has been realized taking into account the characteristics of the physical system to be simulated.

\section{- Loading particles}

In order to provide a precise, noiseless representation of the initial electron density distribution, computational particles are allowed to have different charge. This can be obtained by using the Mass Matrix formulation [16], according to which the particle properties are calculated at the beginning of each simulation.

\section{- Interpolation particle-grid}

The density on the grid is calculated from the particles through the new interpolation scheme defined in Eq. (6). The classic Cloud-In-Cell (CIC) method [15] is used for the interpolation functions. Slightly different expressions are used when a particle approaches the center or the boundary of the domain.

\section{- Poisson solver}

The Poisson equation can be solved with an efficient algorithm based upon the Fast Fourier Transform in $\theta$, assuming a piecewise constant density distribution in $r$ [13]. A set of decoupled differential equations is obtained for the Fourier components $\widetilde{\phi}_{k}(r)$ :

$$
\frac{1}{r} \frac{d}{d r}\left(r \frac{d \widetilde{\phi}_{k}}{d r}\right)+\frac{1}{r^{2}}\left(\frac{\sin \frac{\Delta \theta}{2}}{\frac{\Delta \theta}{2}}\right)^{2} \widetilde{\phi}_{k}=\widetilde{n}_{k}(r) .
$$

Eqs. (7) are solved using a finite difference scheme. Then the velocity field can be calculated by using the central difference discretization.

\section{- Interpolation grid-particle}

Given the velocity field on the grid, the velocity on each particle can be calculated using the same CIC interpolation scheme:

$$
\mathbf{v}_{p}=\sum_{c} \mathbf{v}_{c} w\left(\vec{\omega}_{c}-\vec{\omega}_{p}\right) .
$$

\section{- Particle mover}

Particles are moved solving the equations of motion $d \mathbf{r}_{p} / d t=\mathbf{v}_{p}$ discretized with the fourth-order Runge-Kutta method.

Figures 2 and 3 compare two simulations that only differ in using (6) (in the following we will refer to this code as an Advanced PIC code or KANDINSKY code) or the usual $\rho=\sum_{p} q_{p} w /(r \Delta r \Delta \theta)$ (Standard PIC code) for interpolating the density on the grid. This run has been done for an initial hollow density profile with a $k=2$ perturbation. It can be noticed that very early some differences arise, in particular the Standard PIC code develops some high density vortices (the maximum value of the density almost doubles from the first to the last picture of Fig. 2) while this does not happen for the Advanced PIC code (the maximum of the density remains constant). In the Standard PIC code, we add an artificial compressibility to the system which is manifested by the localized vortices with increasing density.

Furthermore, in the Standard PIC code enstrophy grows because of the contribution of high density vortices, while we would expect enstrophy to be constant (as it is theoretically for the Euler model) or to decrease slowly (because the density develops smaller 

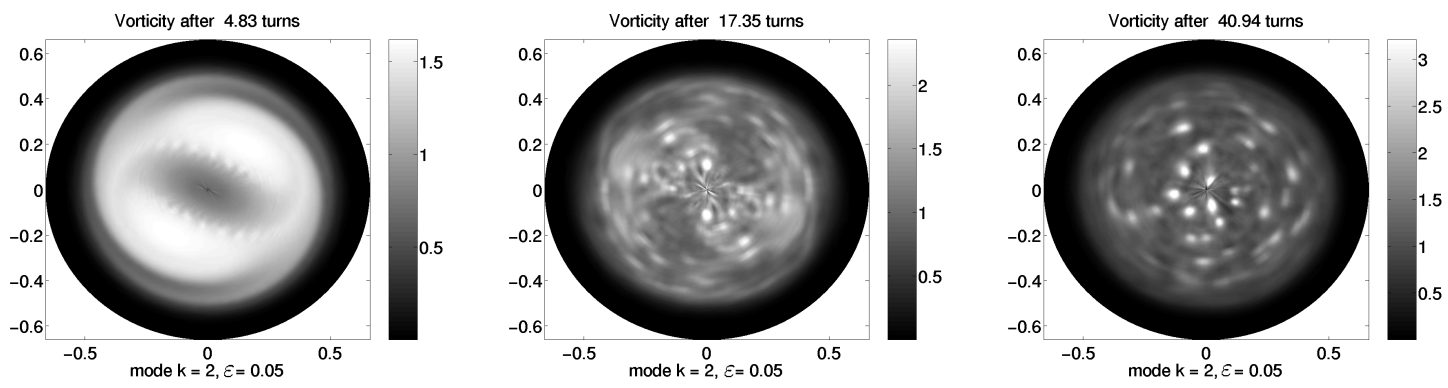

FIGURE 2. Standard PIC Code for a $k=2$ instability in a hollow profile.
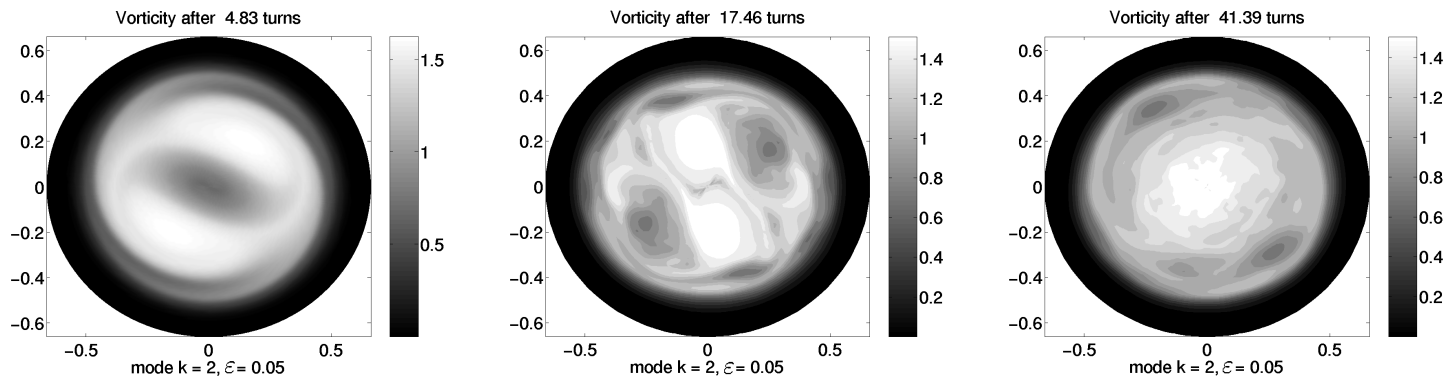

FIGURE 3. KANDINSKY Code for a $k=2$ instability in a hollow profile.

and smaller length scales and at some point it is dissipated by numerical viscosity). Indeed, for the Advanced PIC code, enstrophy is a slowly decreasing function of time.

\section{VALIDATION TESTS}

In this section we use the Euler model, which can be obtained from system (2) by taking $\mathcal{L}_{0}(r)=$ const, in order to compare our results with analytical results and with a reference Eulerian fluid code. In all of our simulations we use an initial equilibrium density profile of the form

$$
n_{c 0}(r)= \begin{cases}n_{0}(0)\left[1-\left(\frac{r}{r_{p}}\right)^{2}\right]^{2}\left[1+(\mu+2)\left(\frac{r}{r_{p}}\right)^{2}\right] & 0 \leq r \leq r_{p} \\ 0 & r>r_{p},\end{cases}
$$

which depends on three parameters: $n_{0}(0), r_{p}$ and $\mu$. The last one is particularly important: for $\mu>0$ the profile is hollow (possible diocotron instability) where for $\mu<0$ the profile is monotonic (always stable). The simulations below are done with $\mu=10$, $r_{p}=0.59$ and $n_{0}(0)$ is specified to give $\int_{0}^{R_{w}} n_{c 0}(r) 2 \pi r d r=1$. The normalized temperature $\alpha$ is chosen equal to 0 . According to [6] the equilibrium profile of $\sigma$ is obtained by $\sigma_{0}=n_{c 0} \mathcal{L}_{0}$. The initial charge density is perturbed by modifying the particle charge as $q_{p}^{\prime}=q_{p}[1+\varepsilon \cos (k \theta)]$, where $k$ is the mode number and $\varepsilon$ is the perturbation amplitude. The system is discretized with a $N_{r} \times N_{\theta}$ grid and using $N_{p}$ particles. In the cases below 

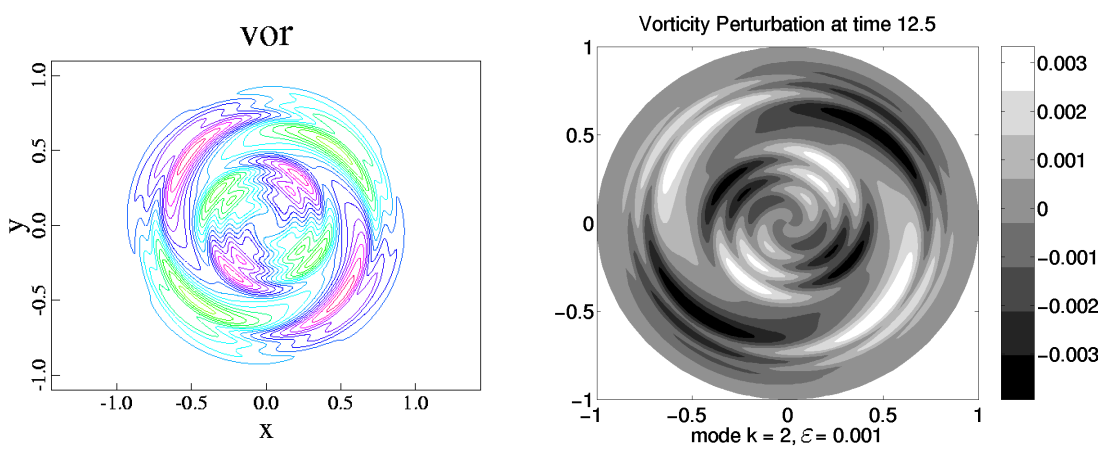

FIGURE 4. Vorticity (density) perturbation from a) Eulerian Fluid Code: linear phase (left); b) KANDINSKY Code: linear phase (right).

we use $N_{r}=150, N_{\theta}=128, N_{p}=558208$, the average number of particles per cell is 49 and the time step is $d t=0.1$.

\section{- Test of accuracy}

Three important conserved quantities are canonical angular momentum, proportional to $P=\iint r^{2} n_{c} r d r d \theta$, electrostatic energy, proportional to $H=\iint n_{c} \phi_{c} r d r d \theta$, and enstrophy, proportional to $Z=\iint n_{c}^{2} r d r d \theta$. (There are additional invariants $\iint f\left(n_{c}\right) r d r d \theta$, with $f$ any function.)

The first two quantities are conserved well by the code (in a run with $k=2, \varepsilon=0.01$ and $t_{\max }=600$, not shown): $\delta P / P=0.6 \%$ and $\delta H / H=0.3 \%$. The enstrophy is weakly conserved due to filamentation, but still the code preserved it well: $\delta Z / Z=5 \%$.

\section{- Comparison with a Reference Eulerian Fluid Code}

The code has been compared with a reference Eulerian fluid code in both the linear and non-linear phase. Figs. $4 \mathrm{a}$ and $4 \mathrm{~b}$ show the evolution of the perturbation of the density at $t=12.5$. The initial perturbation has been chosen very small $(\varepsilon=0.001)$ in order to remain in the linear phase. The agreement between the two codes is remarkable and one can notice that the fluid code has more numerical viscosity than the PIC code.

Figs. 5a and 5b show the evolution of the density after a large initial perturbation $(\varepsilon=0.25)$. The two codes agree very well, even in this non-linear case. It should be noticed that the Eulerian code cannot follow with enough accuracy the region with steep gradients outside the core region.

\section{SIMULATIONS}

In this section, we analyse the dynamics of models (2), (3) and (4). We compare different runs with $k=1$ and $\varepsilon=0.001$. Model (2) can simulate GFD by using a suitable equilibrium length profile, as it will be explained in the following. The shallowwater equation for the potential vorticity can be simplified assuming small topography variations or small variations of the Coriolis parameter. In the last case, $(\xi+2 \Omega) / h$ can be approximated as $\xi+\beta r-\gamma r^{2}$. For $\gamma=0$ we have the $\beta$-plane approximation, while for $\beta=0$ we have the $\gamma$-plane approximation [12]. The analogy between non-neutral plasmas and GFD is established using the linear theory, where $\beta-2 \gamma r$ can be identified with $n_{c 0}(r) \mathcal{L}_{0}^{\prime}(r) / \mathcal{L}_{0}(r)[5,8]$. Moreover, due to the fact that $\mathcal{L}_{0}^{\prime}(0)=0$, it follows that 

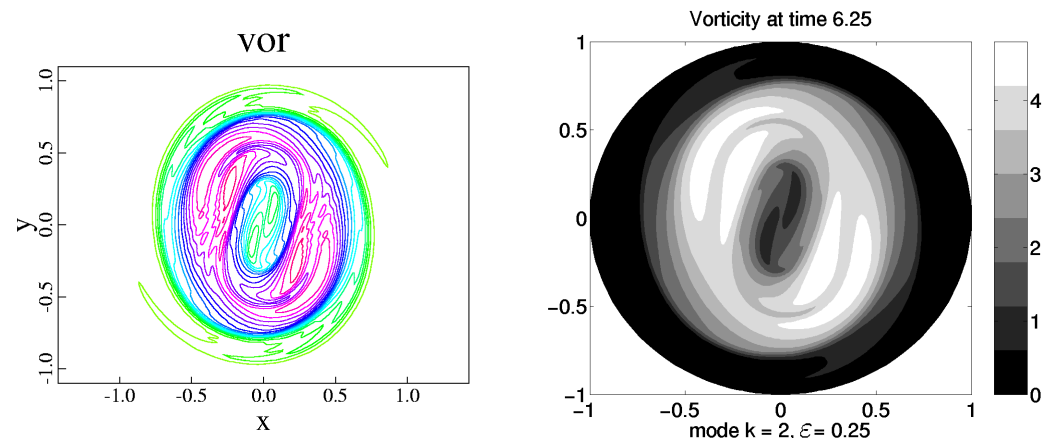

FIGURE 5. Vorticity (density) from a) Eulerian Fluid Code: non-linear phase (left); b) KANDINSKY Code: non-linear phase (right).
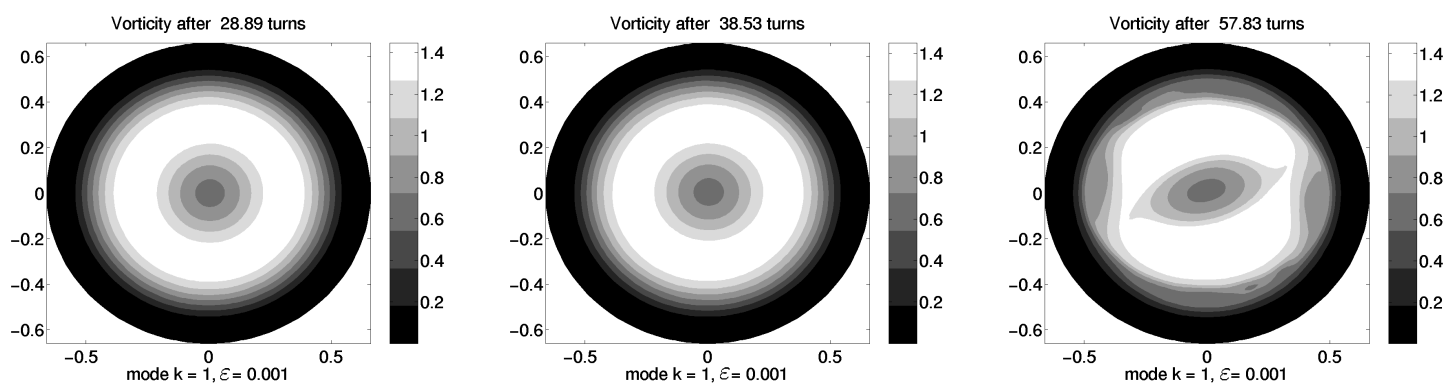

FIGURE 6. Evolution of the density $n_{c}$ according to the Euler model.

$\beta=0$ and thus we are in the limit of the $\gamma$-plane approximation. Therefore, in order to simulate the $\gamma$-plane approximation of GFD, we use model (2) with $\alpha=0$ and the length profile obtained by solving

$$
n_{c 0}(r) \frac{\mathcal{L}_{0}^{\prime}(r)}{\mathcal{L}_{0}(r)}=-2 \gamma r
$$

with the initial density profile given by (9) and $\gamma=s n_{0}(0)$ (this is the value of $\gamma$ obtained from (10) assuming a parabolic profile $\mathcal{L}_{0}=1-s r^{2}$, a constant density profile $n_{c 0}=n_{0}(0)$ and in the limit of small radius, as shown in Ref. [8]). Model (4) is then also simulated in the limit of $\gamma$-plane approximation, using the same $\gamma=s n_{0}(0)$. In all the simulations below we use the curvature $s=0.5$.

Figure 6 shows the evolution of the density for the Euler model after an initial $k=1$ perturbation. The model does not develop any $k=1$ instability and a slowly growing $k=2$ mode eventually dominates. Figure 7 shows the density at the same times according to model (2). This model exhibits a strong $k=1$ diocotron instability which dominates the $k=2$ mode seen in Fig. 6. The low-density core region is pushed off-axis and the high-density region fills the center of the trap. At later times (not shown), the low-density region is redistributed by filamentation or in big vortices. The same picture can be drawn from Fig. 8, which shows the vorticity (density) evolution of the GFD model. The density develops the same features in Figs. 7 and 8 with small differences at later times due to non-linearities.

Clearly, the comparison of Figs. 6, 7 and 8 proves that a Penning trap can simulate GFD and particularly its peculiar features not present in the classic Euler model. 

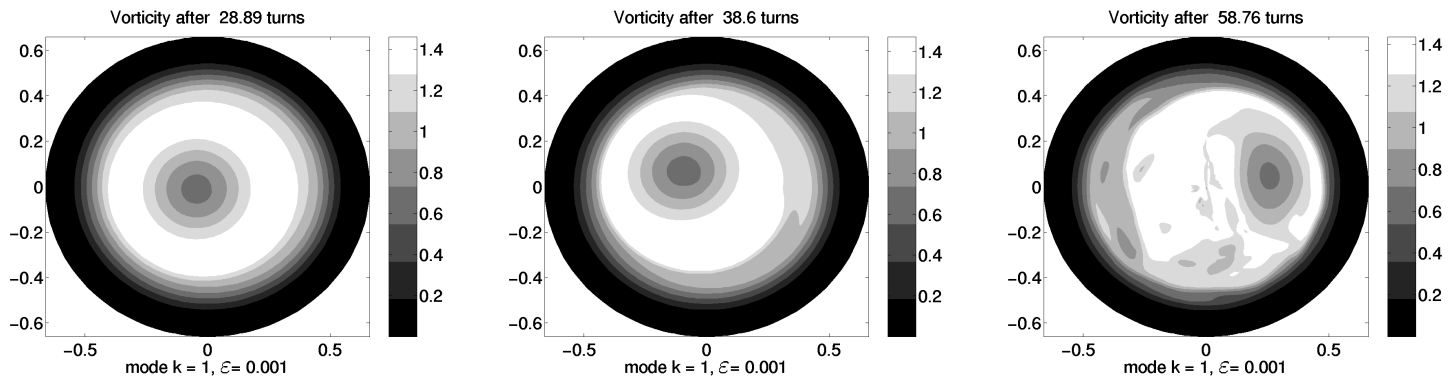

FIGURE 7. Evolution of the density $n_{c}$ according to model (2).
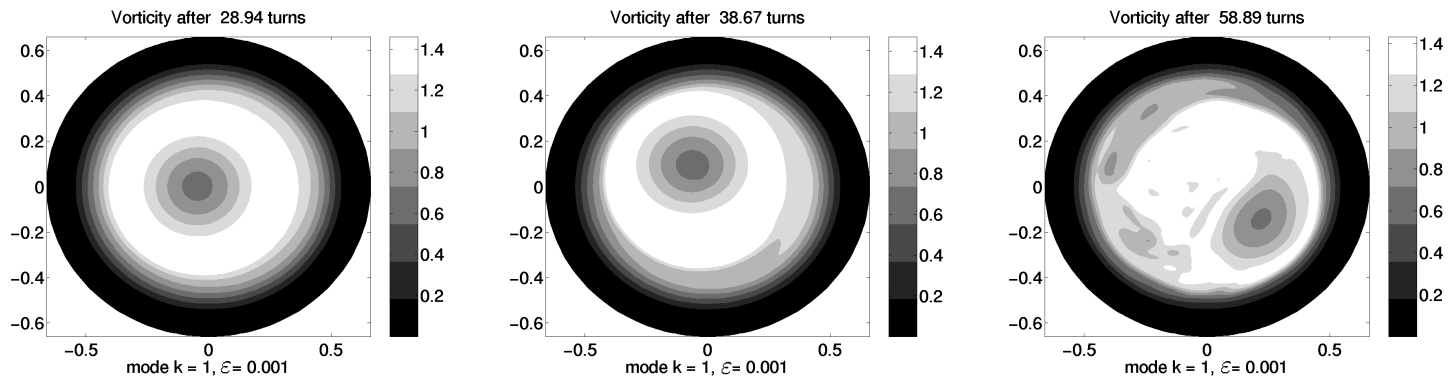

FIGURE 8. Evolution of the vorticity $\xi$ according to the GFD model.

\section{REFERENCES}

1. Briggs, R. J., Daugherty, J. D., and Levy, R. H., Phys. Fluids 13, 421-432 (1970).

2. Davidson, R. C., An Introduction to the Physics of Nonneutral Plasmas, Addison-Wesley, Redwood City, 1990.

3. Smith, R. A., and Rosenbluth, M. N., Phys. Rev. Lett. 64, 649-652 (1990).

4. Driscoll, C. F., and Fine, K. S., Phys. Fluids B 2, 1359-1366 (1990).

5. Finn, J. M., del-Castillo-Negrete, D., and Barnes, D. C., Phys. Plasmas 6, 3744-3758 (1999); Finn,

J. M., del-Castillo-Negrete, D., and Barnes, D. C., Phys. Rev. Lett. 84, 2401-2404 (2000).

6. Coppa, G. G. M., D’Angola, A., Delzanno, G. L., and Lapenta, G., Phys. Plasmas 8, 1133-1141 (2001).

7. Hilsabeck, T. J., and O'Neil, T. M., Phys. Plasmas 8, 407-422 (2001).

8. del-Castillo-Negrete, D., Finn, J. M., and Barnes, D. C., "The modified drift-Poisson model: analogies with geophysical flows and Rossby waves", in 1999 Workshop on Nonneutral Plasmas, edited by J. J. Bollinger, R. L. Spencer, R. C. Davidson, AIP Conference Proceedings 498, New York, 1999, pp. 147152.

9. Dubin, D. H. E., and O'Neil, T. M., Phys. Plasmas 5, 1305-1314 (1998).

10. Coppa, G. G. M., D’Angola, A., Delzanno, G. L., and Lapenta, G., "Rigorous Fluid Model for 3D Analysis of the Diocotron Instability", these proceedings.

11. Delzanno, G. L., Pariev, V. I., Lapenta, G., and Finn, J. M., "Diocotron Spectrum with Compression Effects", these proceedings.

12. Salmon, R., Lectures on Geophysical Fluid Dynamics, Oxford University Press, New York, 1998.

13. Coppa, G. G. M., D’Angola, A., and Lapenta, G., "Simulation of the Evolution of the Diocotron Instability", in 1999 Workshop on Nonneutral Plasmas, edited by J. J. Bollinger, R. L. Spencer, R. C. Davidson, AIP Conference Proceedings 498, New York, 1999, pp.129-134.

14. Brackbill, J. U., and Ruppel, H. M., Jour. of Comp. Phys. 65, 314-343 (1986).

15. Hockney, R. W., and Eastwood, J. W., Computer simulation using particles, Adam Hilger, Bristoll, 1988.

16. Burgess, D., Sulsky, D., and Brackbill, J. U., Jour. of Comp. Phys. 103, 1-15 (1992). 